\title{
Maintenance of dominant populations in heavily grazed grassland: Inference from a Stipa breviflora seed germination experiment
}

\author{
Wenting Liu ${ }^{1}$, Zhijun Wei ${ }^{\text {Corresp., }}{ }^{2}$, Xiaoxia Yang ${ }^{1}$ \\ 1 Qinghai University, Qinghai Academy of Animal and Veterinary Sciences, Xining, China \\ 2 Key Laboratory of Grassland Resources, Ministry of Education P.R. of China, Huhhot, P.R. China, College of Grassland, Resources and Environment, Inner \\ Mongolia Agricultural University, Hohhot, China \\ Corresponding Author: Zhijun Wei \\ Email address: nmndwzj@imau.edu.cn
}

An understanding of population adaptation and maintenance mechanisms under interference from large herbivores is lacking and is a major focus of ecological research. In the Eurasian steppe, which has been subjected to continuous interference from domesticated ungulates throughout history and shows increased grazing, it is particularly urgent to analyse the ecological adaptation strategies of widely distributed Stipa plants. In this study, Stipa breviflora in a group of desert steppes in the Mongolian Plateau was selected to study the potential mechanism underlying the maintenance of dominant populations under the continuous interference of heavy grazing from the new perspective of seed germination rate. Laboratory experimental results showed that the values of the phenotypic traits of $S$. breviflora seeds were lower under a heavy grazing treatment than under a non-grazing treatment, but the seed germination rate did not decrease. The awns of non-grazed seeds significantly affected the seed germination rate, while those of heavily grazed seeds did not. Field observations showed that grazing does not significantly affect the population density of $S$. breviflora at different growth stages except in extremely wet and dry years. Our study suggests that under heavy grazing, S. breviflora uses an "opportunistic" ecological strategy to ensure population maintenance by increasing the seed germination rate and reducing dispersal via changes in associated seed phenotypic traits. 
1 Maintenance of Dominant Populations in Heavily Grazed Grassland: Inference from a Stipa breviflora

2 Seed Germination Experiment

3 Wenting Liu, Zhijun Wei, Xiaoxia Yang

$4 \quad$ Wenting Liu

5 Qinghai University, Qinghai Academy of Animal and Veterinary Sciences, Xining, China.

6 E-mail: qhdxlwt@163.com

7 Zhijun Wei (Corresponding author)

8 Key Laboratory of Grassland Resources, Ministry of Education P.R. of China, Huhhot, P.R. China, College of

9 Grassland, Resources and Environment, Inner Mongolia Agricultural University, Hohhot, China.

10 E-mail: nmndwzj@imau.edu.cn;nmndwzj@163.com

11 Xiaoxia Yang

12 Qinghai University, Qinghai Academy of Animal and Veterinary Sciences, Xining, China.

13 E-mail: yxx@qhmky.com 
14 Abstract: An understanding of population adaptation and maintenance mechanisms under interference from large herbivores is lacking and is a major focus of ecological research. In the Eurasian steppe, which has been subjected to continuous interference from domesticated ungulates throughout history and shows increased grazing, it is particularly urgent to analyse the ecological adaptation strategies of widely distributed Stipa plants. In this study, Stipa breviflora in a group of desert steppes in the Mongolian Plateau was selected to study the potential mechanism underlying the maintenance of dominant populations under the continuous interference of heavy grazing from the new perspective of seed germination rate. Laboratory experimental results showed that the values of the phenotypic traits of $S$. breviflora seeds were lower under a heavy grazing treatment than under a non-grazing treatment, but the seed germination rate did not decrease. The awns of non-grazed seeds significantly affected the seed germination rate, while those of heavily grazed seeds did not. Field observations showed that grazing does not significantly affect the population density of S. breviflora at different growth stages except in extremely wet and dry years. Our study suggests that under heavy grazing, S. breviflora uses an "opportunistic" ecological strategy to ensure population maintenance by increasing the seed germination rate and reducing dispersal via changes in associated seed phenotypic traits.

\section{Introduction}

The Eurasian steppe, which includes a wide distribution of Stipa plants (Gonzalo et al., 2013; Ghiloufi et al., 2016; Nobis et al., 2016; Wieczorek et al., 2017), stretches across Northeast China, Mongolia, Russia, Ukraine and Hungary. Historically, this region has been subjected to continuous interference from domesticated ungulates, and grazing has been increasing, leading to widespread declines in species diversity and above-ground productivity (Bai et al. 2007). However, even in desert steppes with average annual precipitation of less than $200 \mathrm{~mm}$ and long-term overgrazing, it is still possible to find Stipa plants living stably and with relatively high biomass (Wang et al., 2014). Therefore, explaining the ecological strategies adopted by the Stipa genus is essential for the conservation management of plant species and our understanding of species dynamics and maintenance mechanisms.

The selective feeding theory for livestock is obviously unable to explain the survival strategies of plants under severe interference from herbivores. The passive, indiscriminate and repeated feeding of herbivores destroys the vegetative and reproductive organs of plants and causes negative effects such as leaf shortening and narrowing 
41 and cluster shrinking (Díaz et al., 2007). Life history theory posits that plants have substantial flexibility in

42 resource allocation between vegetative and reproductive growth (Miller et al., 2006). To ensure the survival of 43 the plant, the vegetative organs are often favoured at the expense of sexual propagation. Assuming that this 44 compensation continues, the survival of plant species will be difficult. Therefore, we expect that the species that can survive stably will have a set of effective ecological strategies allowing them to overcome this dilemma, and sexual propagation is the key link.

Louault et al. (2005) compared traits in long-term grazed grassland with those in non-grazed grassland and found that grazing caused a series of effects, such as a reduction in seed size and a decrease in seed weight. The size of the seed directly affects its scattering, spread and survival (Chambers, 1995). Compared with large-sized seeds, small-sized seeds are able to disperse farther, thereby improving their fitness (Coomes \& Grubb, 2003). Studies have found that the awn of Stipa seeds can help them spread more efficiently. For example, the pubescence on the awns erects when the air is dry to increase the aerostatic buoyancy (Peart, 1981), which reduces the speed at which the seed falls (Greene \& Johnson, 1993); in addition, the pubescence on the awns can attach to the fur of animals to spread the seed. When the propagule falls to the ground, the moisture absorption by the awn allows the seed to move a short distance on the soil surface and complete the self-burial process, allowing the propagule to enter the soil (Elbaum et al., 2007; Jung et al., 2014; Liu et al., 2018a). All of the abovementioned studies suggest the importance of awns for the spread of seeds. There are two interesting conjectures. (Conjecture 1) Awns can inhibit the germination of seeds. Assume that the dispersal process of seeds has not been completed, and seed germination occurs because of favourable temperature, humidity, etc. This seed germination strategy is obviously not ideal. (Conjecture 2) Under heavy grazing, awns cannot effectively inhibit seed germination to prevent unfavourable seed germination and increase the chance of population regeneration.

Desert grassland, accounting for $39 \%$ of the total grassland area in northern China, is the most arid grassland ecosystem on the Eurasian steppe (Li et al., 2000). This grassland is an important part of the semi-arid grassland area of northern China and has a long history of animal husbandry (Wang et al., 2017a). Compared with other grassland types, desert grassland has a low plant species richness and primary productivity due to drought caused by limited rainfall (Li et al., 2008). Therefore, it is valuable to analyse the survival strategies of the dominant 
68

69

70

71

72

73

74

species of the desert steppe that have been overgrazed. Therefore, this study aims to examine the following by focusing on Stipa breviflora in the desert grassland in northern China: 1) the effect of grazing on the morphological characteristics of seeds; 2) the effect of awns on seed germination under grazing and non-grazing conditions; and 3) the interannual variability in plant population density in grazing and non-grazing treatments.

\section{Materials and Methods}

\section{Study Area}

The experimental area is located in the Inner Mongolia Plateau S. breviflora positioning station of Inner Mongolia Agricultural University, Zhurihe Town (E112 47'16.9", N42 $\left.16^{\prime} 26.2^{\prime \prime}\right)$, Xilin (Fig. 1), with an elevation of $1100-1150 \mathrm{~m}$. Here, rain and hot temperatures occur in the same period, with average annual precipitation of $183.0 \mathrm{~mm}$, annual average temperature of $5.8^{\circ} \mathrm{C}$, average annual sunshine hours of $3137.3 \mathrm{~h}$, average annual evaporation of $2793.4 \mathrm{~mm}$, and average annual frost-free period of 177 days. The area is characterized by a medium-temperate climate. The average wind speed is $5.1 \mathrm{~m} / \mathrm{s}$, and the wind is mostly concentrated in winter and spring and dominated by wind from the northwest. The average number of windy days is 67 , and dust storms frequently occur. The soil is light chestnut soil with a deserted surface, and the humus layer is $20-30 \mathrm{~cm}$ thick. The grass layer is low, generally $10-25 \mathrm{~cm}$ in height, and has a low coverage of $15-25 \%$. The vegetation is composed of S. breviflora (cluster of a perennial grass); Cleistogenes songorica and Allium polyrhizum are the dominant plants.

\section{Experimental Design}

The grazing trial began in 2010, starting in May and ending in late October each year. During the experimental period, continuous grazing was adopted. In the evening, the flock was allowed in the plot, with no supplementary feeding. The test plots selected in this study are located in the same continuous section with flat terrain and a relatively uniform environment, effectively controlling for differences in background, terrain and spatial heterogeneity. There were two treatments in this study, namely, the heavy grazing and non-grazing treatments, each with three replicates, and each test plot had an area of 2.60 ha. The heavy grazing treatment had a stocking capacity of 3.08 sheep $\cdot \mathrm{ha}^{-1} \cdot \mathrm{a}^{-1}$, and the non-grazing treatment excluded interference from humans, livestock and large wild animals (Liu et al., 2018b). The herbivores in the grazing area were Sunite sheep that had generally the same health status, individual size, weight and sex. 


\section{Seed Germination Experiment}

96 Since the seeds of Stipa undergo a period of dormancy after maturation (Zhang et al., 2017), this study attempts

97 to explore the ecological strategies of S. breviflora populations under grazing from the perspective of seed 98 germination. To minimize the effect of seed dormancy, we chose the period when the germination rate of $S$. breviflora seeds was the highest. Then, on June 3, 2015, in each experimental plot, mature reproductive shoots of strong $S$. breviflora plants with a high seed-setting rate were selected. After being air dried, the seeds were manually threshed and stored in a bag for winter use. On June 15, 2016, the seeds were brought back to the laboratory, and the seeds from the same treatment were uniformly mixed. Fifty seeds were randomly selected for each grazing treatment, and the seed width, seed length, seed weight, and awn weight were measured with Vernier callipers.

1) Seeds with superior morphological characteristics were selected, rinsed with tap water, sterilized with $75 \%$ ethanol, rinsed with deionized water, and treated with awning and non-awning treatments. The seeds were then placed in twenty 9-cm-diameter Petri dishes, with 50 seeds per dish. Each treatment was repeated 5 times. The light and dark periods were each $12 \mathrm{~h}$, and the dishes were kept at $25^{\circ} \mathrm{C}$ in a constant temperature incubator. The germination status of the $S$. breviflora seeds in each dish was recorded every $24 \mathrm{~h}$. An appropriate amount of water was added after recording the germination status to ensure consistent processing conditions. The assay was terminated after 14 days (Liu et al., 2018a) (Fig. 1A).

2) To assess whether the seeds of S. breviflora were dormant, a low-temperature treatment method was used in this study as well as in preliminary and related studies (Wang et al., 2017b). The seeds from the heavy grazing treatment and non-grazing treatment were placed in a refrigerator at $4{ }^{\circ} \mathrm{C}$ for the low-temperature treatment. After 4 weeks of the low-temperature treatment, all seeds were removed, rinsed with distilled water and then germinated. Step 1 was repeated for the germination test (Fig. 1B).

3) To analyse the potential factors affecting the germination rate (germination percentage) of $S$. breviflora seeds, we added the awns, seeds, and seeds + awns, which were heavily grazed, to the culture medium for the germination test of non-grazed seeds, whose awns, seeds, and seeds + awns were separately added to the culture medium for the germination test of heavily grazed seeds. Specifically, 50 heavily grazed S. breviflora seeds 
122 covered with damp blotting paper. The production of heavily grazed awn (HGA), heavily grazed seed + awn

123 (HGT), non-grazed seed (NGS), non-grazed awn (NGA), and non-grazed seed + awn (NGT) additives was

124 completed as described above. The seed germination test without additives was set as a control treatment (CK),

125 and each treatment was repeated 5 times. Fifty heavily grazed seeds were placed in the medium where NGS

126 additives had been added, and 50 NGSs were added to the medium where heavily grazed seed additives had been

127 added; an appropriate amount of distilled water was then supplied. Step 1 was repeated for the germination test

128 (Fig. 1C).

4) To further verify the factors affecting the germination rate of S. breviflora seeds, this study selected cabbage seeds as the test object and used the six types of additives described above to perform germination tests (Bao et al., 2015). Germination experiments without any additives were set as controls, and each treatment was repeated 5 times. Fifty grazed cabbage seeds were placed in each medium, and the seed germination process was carried out with the abovementioned germination test procedure (Step 1) (Fig. 1D).

\section{4}

135

\section{Survey of the S. breviflora Population}

During the period of peak plant growth from 2010 to August 2017, five sample plots, each with an area of $1 \times 1$ $\mathrm{m}^{2}$, were randomly selected in each test plot to determine the number of $S$. breviflora individuals. According to the measured basal diameter (Bd) of S. breviflora and a previous study (Liu et al., 2017), S. breviflora was divided into five stages based on age: seedling stage ( $\mathrm{Bd} \leq 4 \mathrm{~mm})$, juvenile stage ( $4 \mathrm{~mm}<\mathrm{Bd} \leq 20 \mathrm{~mm}$ ), adult stage $(20 \mathrm{~mm}<\mathrm{Bd} \leq 40 \mathrm{~mm})$, pre-ageing stage $(40 \mathrm{~mm}<\mathrm{Bd} \leq 70 \mathrm{~mm})$, and ageing stage $(\mathrm{Bd}>70 \mathrm{~mm})$. From 2015 to the first ten-day period of August 2017, five sample plots, each with an area of $1 \times 1 \mathrm{~m}^{2}$, were randomly selected in each test plot to investigate the number of $S$. breviflora individuals at each growth stage.

\section{Meteorological Data}

Meteorological data were obtained from a micro weather station (GroWeather ${ }^{\circledR}$ software version 1.2, Davis Instruments Corporation, USA) located at the experimental site (Wang et al., 2014).

\section{Statistical Analysis}

An independent t-test was conducted to study the seed width, length and weight; awn weight; germination rate of seeds with awns and those without awns as well as the germination rate of seeds with awns and those without awns after the non-grazing treatment under the heavy grazing and non-grazing treatments. One-way analysis of 
variance was performed to compare the NGSs, NGAs, NGTs and germination rates of heavily grazed $S$. breviflora seeds with the CK, and Duncan's test was used for multiple comparisons. Furthermore, one-way analysis of variance was performed to compare the HGSs, HGAs, HGTs and germination rates of non-grazed $S$. breviflora seeds with the CK, and Duncan's test was used for multiple comparisons. Additionally, the NGSs, NGAs, NGTs, HGSs, HGAs, HGTs and seed germination rates of Chinese cabbage (CK) were analysed, and Duncan's test was used for multiple comparisons. An independent t-test was adopted to compare the density ratio of $S$. breviflora and the density of $S$. breviflora between the heavy grazing treatment and non-grazing treatment in the same year. The statistical analyses described above were completed in SAS 9.0 (SAS Institute Inc., Cary NC, USA).

\section{Results}

\section{Seed Phenotypic Traits and Germination Rate}

The results of the independent t-test (Fig. 2) showed that the values of the phenotypic traits of non-grazed $S$. breviflora seeds were significantly higher than those of heavily grazed $S$. breviflora seeds $(\mathrm{P}<0.001)$; specifically, the seed length, width, and weight and awn weight were $10.47 \%, 13.60 \%, 36.02 \%$, and $41.85 \%$ higher, respectively. The germination rate of non-grazed $S$. breviflora seeds with no awns was $386.96 \%$ higher than that of such seeds with awns $(\mathrm{P}<0.001)$, while there was no significant difference in the germination rate between the two types of seeds under the heavy grazing treatment $(\mathrm{P}>0.05)$ (Fig. 3A). The germination rate of heavily grazed seeds with awns was significantly higher than that of NGSs with awns. However, there was no significant difference in the germination rate of seeds with no awns between the heavy grazing treatment and the non-grazing treatment.

\section{Impact of Awns on Seed Germination Rate}

Regarding whether the germination rate of $S$. breviflora seeds is affected by seed dormancy, this study found that the germination rate was the same before and after dormancy release by physically breaking dormancy. Specifically, under the non-grazing treatment, seeds with awns had a significantly lower germination rate than those without awns, and the germination rate of seeds without awns was not obviously different between the heavy grazing treatment and the non-grazing treatment (Fig. 3B).

The results of the tests with additives showed (Fig. 4) that S. breviflora seeds under the heavy grazing treatment 
176 in the culture medium with the NGA and NGT additives had a significantly lower germination rate than seeds

177 with no additive $(\mathrm{CK})(\mathrm{P}<0.05)$, while the germination rate of those with NGS additives did not significantly

178 differ from that of seeds with no additive $(\mathrm{CK})(\mathrm{P}<0.05)$ (Fig. 4A). In the culture medium of non-grazed $S$.

179 breviflora seeds, the germination rate with the NGS additive was significantly higher $(\mathrm{P}<0.05)$ than that with

180 the NGA additive under heavy grazing, but there was no significant difference in the germination rate between

181 those with the NGT additive and those without any additive (Fig. 4B).

182 To avoid the regulation of $S$. breviflora seed germination by their own substances and further verify that awns

183 affect the germination of $S$. breviflora seeds, this study used cabbage seeds as a test object. The germination rate

184 of Chinese cabbage seeds with the awn additive was significantly lower than that of the CK, and that of cabbage

185 seeds with the NGA additive was $49.10 \%$ lower than that under the heavy grazing treatment $(\mathrm{P}<0.05)($ Fig. 5$)$.

186 Dynamics of S. breviflora Populations

187 The 2010-2017 field survey results (Fig. 6) showed that in all years except 2014 and 2016 , there was no

188 significant difference in the ratio of the density of heavily grazed S. breviflora plants to the number of plants in

189 the community and that of non-grazed S. breviflora plants to the number of plants in the community $(\mathrm{P}>0.05)$.

190 Further analysis revealed that there was also no significant difference in the density and density ratios of $S$.

191 breviflora at different growth stages between the heavy grazing and non-grazing treatments in 2015 and 2017 ,

192 and in 2016, the S. breviflora seedlings and juveniles in the non-grazing treatment showed a significantly higher

193 density than those in the heavy grazing treatment, with no difference displayed at any of the other growth stages

194 (Fig. 7).

195 Discussion

196 Chen et al. (2017) found that heavy grazing affects the morphological characteristics of grassland plant seeds, 197 and this finding is consistent with the findings of this study. Specifically, compared with the non-grazing treatment, heavy grazing significantly reduced the seed length, seed width, seed weight, and awn weight of $S$. breviflora on the desert steppe. This finding may be because grazing reduced the number of reproductive individuals in the S. breviflora population (Liu et al., 2018a). Moreover, although the plants have substantial flexibility in resource allocation between growth and reproduction (Miller et al., 2006), their vegetative and 
203

204

205

206

207

208

209

210

211

212

213

214

215

216

217

218

219

220

221

222

223

224

225

226

227

228

229

feeding by livestock. When plants have a constant total amount of resources and a limited ability to assimilate resources, they have to invest more resources to produce vegetative organs, compensating for the grazinginduced damage at the expense of sacrificing sexual reproduction (Fornara \& Du Toit, 2007) and causing a series of reactions such as the seed shortening, narrowing, and weight reduction observed in S. breviflora. A study in the Mediterranean steppe further validated our conjecture. When the stocking capacity is greater than 3 sheep $\cdot$ ha $^{-}$

${ }^{1}$, the native plant species show a significant increase in vegetative growth and a reduction in reproductive growth (De Miguel et al., 2010). This pattern indicates that to some extent, phenotypic trait changes are a comprehensive manifestation of plant populations adapting to changes in the external environment (Louault et al., 2005).

Although heavy grazing alters the morphological characteristics of seeds, it is interesting to note that heavily grazed S. breviflora seeds (without an awn) generally exhibit the same germination rate as NGSs (without an awn); that is, the germination rate of large seeds does not differ from that of small seeds, which is contrary to the results of Zimmerman and Weis (1983), Hendrix and Trapp (1992), and Greipsson and Davy (1995). We assume that in response to heavy grazing, S. breviflora has formed its own unique survival strategy over its longterm interaction with livestock: S. breviflora can carry out both sexual reproduction and asexual reproduction. However, it is obviously not favourable to increase the number of asexually reproducing individuals under severe interference from livestock because individuals who vegetatively propagate seedlings usually form small-scale aggregations with the mother plant, which will undoubtedly reduce the feeding cost of livestock and is not conducive to the survival of the population. Compared to large seeds (no grazing), at the same wind speed, awns can help smaller seeds (heavy grazing) disperse farther (Matlack, 1987), and this increase in dispersal is more propitious for the spread and colonization of the population. However, there is an obvious problem. If the seed can spread farther but has a lower germination rate, then further dispersal obviously has no biological significance. This condition means that the seeds of heavily grazed plants need a higher germination rate. Our previous findings provide the basis for this hypothesis. Grazing can disrupt the threshold for germination of $S$. breviflora seeds; that is, the weights of germinable seeds under a heavy grazing treatment were $25 \%$ lower than those under a non-grazing treatment (Liu et al., 2018a). The results of this study also showed that grazing does not affect the germination rate of S. breviflora seeds (Fig. 3), suggesting that the high germination rate of heavily grazed seeds may be an important mechanism for population maintenance. 
230

231

232

233

234

235

236

237

238

239

240

241

242

243

244

245

246

247

248

249

250

251

252

253

254

255

256

Additionally, the germination rate of heavily grazed S. breviflora seeds (with awns) was significantly higher than that of NGSs (with awns), and the germination rate of awn-free seeds was significantly higher than that of seeds with awns. This result indicates that awns can dramatically affect the germination rate of $S$. breviflora seeds. Numerous studies have suggested that rapid seed germination is a common survival mechanism for plants in arid regions (Wallace et al., 1968), but our results do not fully support this view. This discrepancy probably occurs because the desert grassland is dry and rainless, and effective rainfall can promote not only large-scale seed germination but also the growth and development of individual plants. In the non-grazing treatment, with relatively good plant growth, this increase in performance due to rainfall will undoubtedly increase the competitiveness within and among plant species and cause the death of $S$. breviflora seedlings that are poor competitors, which is not conducive to the regeneration and survival of the population. Furthermore, awns can help seeds disperse over long distances. If seeds germinate before they are dispersed, then this trait is obviously not propitious for the colonization and reproduction of the population. Garnier and Dajoz (2001) also confirm our view, as the germination of Hyparrhenia diplandra seeds was not observed during the dispersal process in savannas. All of the abovementioned findings indicate that awns can reduce the germination rate of $S$. breviflora seeds, especially those not subjected to grazing (Fig. 5). Therefore, we believe that the awn of the propagule may play an extremely important role in the reproduction of plants. Once the seeds are mature, the propagule is detached from the spikelets by the twisting of the awns (Raju \& Ramaswamy, 1983), and the population is able

to spread with the help of wind or animals. In this process, the presence of the awn columns inhibits the germination of the seeds and prevents germination from occurring under unfavourable conditions.

However, in the heavy grazing treatment, although the germination rate of seeds without awns was $22.90 \%$ higher than that of seeds with awns, the awns did not significantly affect the germination rate of $S$. breviflora seeds (Fig. 3). This result seems to contradict the aforementioned result, but we believe that this pattern reflects a series of behavioural mechanisms for plants to adapt to herbivores. The change in awns is highly heritable (Garnier and Dajoz, 2001). In the case of heavy grazing, due to long-term, intense feeding of livestock, individual plants become sparse, the competition within and among species is weakened, the exposed land area is increased, and the water holding capacity is reduced. Assuming awns effectively inhibited seed germination at this time, this would undoubtedly exacerbate the decline in the population. Therefore, under heavy grazing conditions, the 
257 presence of awns not only provides more favourable sites for the survival of new individuals but also does not

258 force the seeds to germinate after awning, which greatly reduces the time required for seed germination and

259 creates greater odds for the survival of the S. breviflora population.

260 Based on the population density of $S$. breviflora expressed as a proportion of the number of plants in the 261 community in 2010-2017, our research verifies the abovementioned theory to a certain extent; that is, there was

262 no significant difference between the heavy grazing treatment and non-grazing treatment (except in 2014 and 263 2016). Oesterheld \& Sala (1990) also found that grazing increases the number of individuals of dominant species.

264 This result occurs because, in addition to the seeds themselves, livestock have a high ability to spread seeds 265 (Rosenthal et al. 2012), create more favourable habitat opportunities for seed germination, and reduce individual 266 damage caused by competition within or between species. This phenomenon suggests that compared to the $S$. 267 breviflora population under the non-grazing treatment, the heavy grazing treatment adopts a mechanism of 268 accelerating the population renewal rate to maintain the relative stability of the population size. Further analysis found that in 2015 and 2017, there was no significant difference in the number and proportion of $S$. breviflora

270 individuals between the heavily grazed and non-grazed treatments. In arid and rainless desert steppes, extreme 271 precipitation events during the growing season may be triggers that impact the number of dominant populations.

272 Consistent with this pattern, in 2014 (precipitation in May-July = $51.7 \mathrm{~mm}$ ) and 2016 (precipitation in May-July $273=122 \mathrm{~mm}$ ), the population of heavily grazed $S$. breviflora represented a smaller proportion of the plant 274 community than that of non-grazed S. breviflora, and in 2016, the density of non-grazed S. breviflora seedlings and juveniles was significantly higher than that of heavily grazed S. breviflora seedlings and juveniles.

This study explored the potential maintenance mechanism of dominant populations from a new perspective of seed germination rate. However, unfortunately, the chemical composition changes of awns under different treatments were not analysed, which means that the biochemical perspective was not included. Additionally, the density of $S$. breviflora individuals at different growth stages was not investigated from the beginning of this study, but from 2015; thus, the typical drought year of 2014 was missed.

\section{Conclusion}

282 The results suggested that heavy grazing reduced the phenotypic characteristics of S. breviflora seeds but did not significantly reduce the seed germination rate, and the awns did not inhibit seed germination. Compared with 
284

285

286

287

288

289

290

291

292

293

294

295

296

297

298

299

300

301

302

303

304

305

306

307

308

309

310

the no grazing and heavy grazing treatments, S. breviflora seeds made full use of the spreading functions of awns and adopted the ecological strategies of maintaining the seed germination percentage and "opportunism" to ensure population maintenance.

\section{Acknowledgements}

We thank Guopeng Liu for his detailed graph (Fig. 1). We also gratefully acknowledge the students from Inner Mongolia Agriculture University for their help with this work.

References

Bai YF, Wu JG, Pan QM, Huang JH, Wang QB, Li FS, Buyantuyev A, Han XG. 2007. Positive linear relationship between productivity and diversity: evidence from the Eurasian steppe. Journal of Applied Ecology 44: 1023-1034 DOI: 10.1111/j.1365-2664.2007.01351.x

Bao HC, Hao LZ, Zhang FL, Yang FL, Yang ZR, Li XL, Zheng QL. 2015. Allelopathic Effects of Aqueous Extracts of Pugionium cornutum on Seed Germination and Seedling Growth of Cabbage. Plant Physiology Journal 51: 1109-1116 DOI: 10.13592/j.cnki.ppj.2015.0134

Chambers JC. 1995. Relationships between Seed Fates and Seedling Establishment in an Alpine Ecosystem. Ecology 76:2124-2133 DOI: 10.2307/1941687

Chen T, Christensen M, Nan Z, Hou FJ. 2017. Effects of grazing intensity on seed size, germination and fungal colonization of Lespedeza davurica, in a semi-arid grassland of northwest China. Journal of Arid Environments 144: 91-97 DOI: 10.1016/j.jaridenv.2017.04.006

Coomes DA, Grubb PJ. 2003. Colonization, tolerance, competition and seed-size variation within functional groups. Trends in Ecology \& Evolution 18: 283-291 https://doi.org/10.1016/S0169-5347(03)00072-7

Díaz S, Lavorel S, McIntyre S, Falczuk V, Casanoves F, Milchunas DG, Skarpe C, Rusch G, Sternberg M, Noy-Meir I, Landsberg J, Zhang W, Clark H, Campbell BD. 2007. Plant trait responses to grazing - a global synthesis. Global Chang Biology 13: 313-341 https://doi.org/10.1111/j.13652486.2006.01288.x

Elbaum R, Zaltzman L, Burgert I, Fratzl P. 2007. The Role of Wheat Awns in the Seed Dispersal Unit. Science 316: 884-886 DOI: $10.1126 /$ science. 1140097

Fornara DA, Du Toit JT. 2007. Browsing lawns? Responses of Acacia nigrescens to ungulate browsing in an 
African savanna. Ecology 88: 200-209 DOI: 10.1890/0012-9658(2007)88[200:BLROAN]2.0.CO;2

Garnier LKM, Dajoz I. 2001. Evolutionary significance of awn length variation in a clonal grass of fire-prone savannas. Ecology 82: 1720-1733 DOI: 10.2307/2679813

Ghiloufi W, Büdel B, Chaieb M. 2016. Effects of biological soil crusts on a Mediterranean perennial grass (L.). Plant Biosystems 151:1-10 DOI: 10.1080/11263504.2015.1118165

Gonzalo R, Aedo C, García MÁ. 2013. Taxonomic Revision of the Eurasian Stipa Subsections Stipa and Tirsae (Poaceae). Systematic Botany 38: 334-378 DOI: 10.1600/036364413X666615

Greene D F, Johnson E A. 1993. Seed Mass and Dispersal Capacity in Wind-Dispersed Diaspores. Oikos 67:6974 DOI: $10.2307 / 3545096$

Greipsson S, Davy AJ. 1995. Seed mass and germination behavior in populations of the dune-building grass Leymus arenarius. Annals of Botany 76: 493-501 doi:10.1006/anbo.1995.1125

Hendrix SD, Trapp EJ. 1992. Population demography of Pastinaca sativa (Apiaceae): Effects of seed mass on emergence, survival, and recruitment. American Journal of Botany 79: 365-375 doi:10.2307/2445148

Jung W, Kim W, Kim HY. 2014. Self-burial mechanics of hygroscopically responsive awns. Integrative and Comparative Biology 54: 1034-1042 DOI: 10.1093/icb/icu026

Li C, Hao X, Zhao M, Han G, Willms WD. 2008. Influence of historic sheep grazing on vegetation and soil properties of a Desert Steppe in Inner Mongolia. Agriculture Ecosystems \& Environment 128: 109-116 DOI: 10.1016/j.agee.2008.05.008

Li SG, He ZY, Chang XL, Harazono Y, Oikawa T, Zhao HL. 2000. Grassland Desertification by Grazing and Resulting Micrometeorological Changes in Inner Mongolia. Agricultural and Forest Meteorology 102: 125-137 DOI: 10.1016/S0168-1923(00)00101-5

Liu WT, Lü SJ, Wang TL, Yan RR, Wei ZJ. 2018b. Large herbivore-induced changes in phytogenic hillocks: links to soil and windblown sediment on the desert steppe in China. Ecological research 33: 889-899 DOI: $10.1007 / \mathrm{s} 11284-018-1582-4$

Liu WT, Wang TL, Zhang S, Ding LJ, Lü SJ, Wei ZJ. 2017. Effects of grazing on edificators and soil aggregate characteristics in Stipa breviflora desert steppe. Ecology and Environmental Sciences 26: 978984 DOI: 10.16258/j.cnki.1674-5906.2017.06.010 
Liu WT, Wang TL, Zhang S, Ding LJ, Wei ZJ. 2018a. Grazing influences Stipa breviflora seed germination in desert grasslands of the Inner Mongolia Plateau. PeerJ 6: e4447 DOI: 10.7717/peerj.4447

Louault F, Pillar VD, Garnier E, Soussana JF. 2005. Plant Traits and Functional Types in Response to Reduced Disturbance in a Semi-Natural Grassland. Journal of Vegetation Science 16: 151-160 DOI: 10.1111/j.1654-1103.2005.tb02350.x

Oesterheld M, Sala OE. 1990. Effects of grazing on seedling establishment: the role of seed and safe - site availability. Journal of Vegetation Science 1: 353-358 DOI: 10.2307/3235711

Matlack GR. 1987. Diaspore size, shape, and fall behaviour in wind-dispersed plant species. American Journal of Botany 74:1150-1160. DOI: 10.1002/j.1537-2197.1987.tb08729.x

De Miguel JM, Casado MA, Pozo AD, Ovalle C, Moreno-Casasola P, TraviesoBello AC, Barrera M, Ricardo N, Tecco PA, Acosta B. 2010. How reproductive, vegetative and defensive strategies of Mediterranean grassland species respond to a grazing intensity gradient. Plant Ecology 210: 97-110 DOI: $10.1007 / \mathrm{s} 11258-010-9741-\mathrm{x}$

Miller TE, Tyre AJ, Louda SM. 2006. Plant reproductive allocation predicts herbivore dynamics across spatial and temporal scales. American Naturalist 168: 608-616 DOI: 10.1086/509610

Nobis M, Klichowska E, Nowak A, Gudkova PD. 2016. Multivariate morphometric analysis of the Stipa turkestanica group (Poaceae: Stipa sect. Stipa). Plant Systematics \& Evolution 302: 137-153 DOI: 10.1007/s00606-015-1243-9

Peart MH. 1981. Further experiments on the biological significance of the seed-dispersal units in grasses. Journal of Ecology 69: 425-436 DOI 10.2307/2259677

Raju MVS, Ramaswamy SN. 1983. Studies on the inflorescence of wild oats(Avena fatua). Canadian Journal of Botany 61:74-78 DOI 10.1139/b83-006.

Rosenthal G, Schrautzer J, Eichberg C. 2012. Low-intensity grazing with domestic herbivores: a tool for maintaining and restoring plant diversity in temperate Europe. Tuexenia 32:167-205

Wallace A, Rhods WA, Frolich EF. 1968. Germination behaviour of Salsola as influenced by temperature, moisture, depth of planting, and gamma irradiation. Agronomy Journal 60:76-78 DOI 10.2134/agronj1968.00021962006000010024x. 
365

366

367

368

369

370

371

372

373

374

375

376

377

378

379

380

381

Wang G, Baskin CC, Baskin J M, Yang X, Liu G, Zhang X, Ye X, Huang Z. 2017b. Timing of seed germination in two alpine herbs on the southeastern Tibetan plateau: the role of seed dormancy and annual dormancy cycling in soil. Plant and Soil 421: 465-476 DOI: 10.1007/s11104-017-3400-0

Wang H, Dong Z, Guo J, Li H, Li J, Han G, Chen X. 2017a. Effects of grazing intensity on organic carbon stock characteristics in Stipa breviflora desert steppe vegetation soil systems. Rangeland Journal. 39: 169177 DOI: $10.1071 / \mathrm{RJ} 16007$

Wang Z, Jiao S, Han G, Zhao M, Ding H, Zhang X, Wang X, Ayers EL, Willms WD, Havsatad K, Lata A, Liu Y. 2014. Effects of stocking rate on the variability of peak standing crop in a desert steppe of Eurasia grassland. Environmental Management 53: 266-273 DOI: 10.1007/s00267-013-0186-6

Wieczorek K, Bugaj-nawrocka A, Kanturski M, Miller GL. 2017. Geographical variation in morphology of Chaetosiphella stipae Hille Ris Lambers, 1947 (Hemiptera: Aphididae: Chaitophorinae). Scientific Reports 7:43988 DOI: $10.1038 /$ srep43988

Zhang R, Baskin JM, Baskin CC, Mo Qing, Chen LJ, Hu XW, Wang YR. 2017. Effect of population, collection year, after-ripening and incubation condition on seed germination of Stipa bungeana. Scientific Reports 7:13893 DOI:10.1038/s41598-017-14267-2

Zimmerman JK, Weis IM. 1983. Fruit size variation and its effects on germination and seedling growth in Xanthium strumarium. Canadian Journal of Botany 61: 2309-2315 DOI:10.1139/b83-253 
Figure 1

Seed germination test process. NAT denotes normal atmospheric temperature 
A

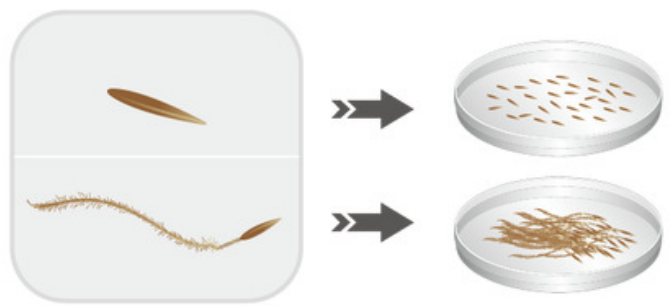

B
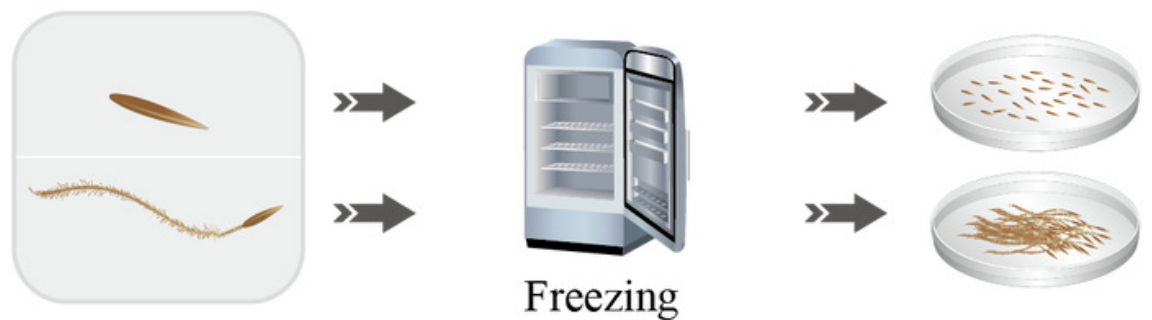

C
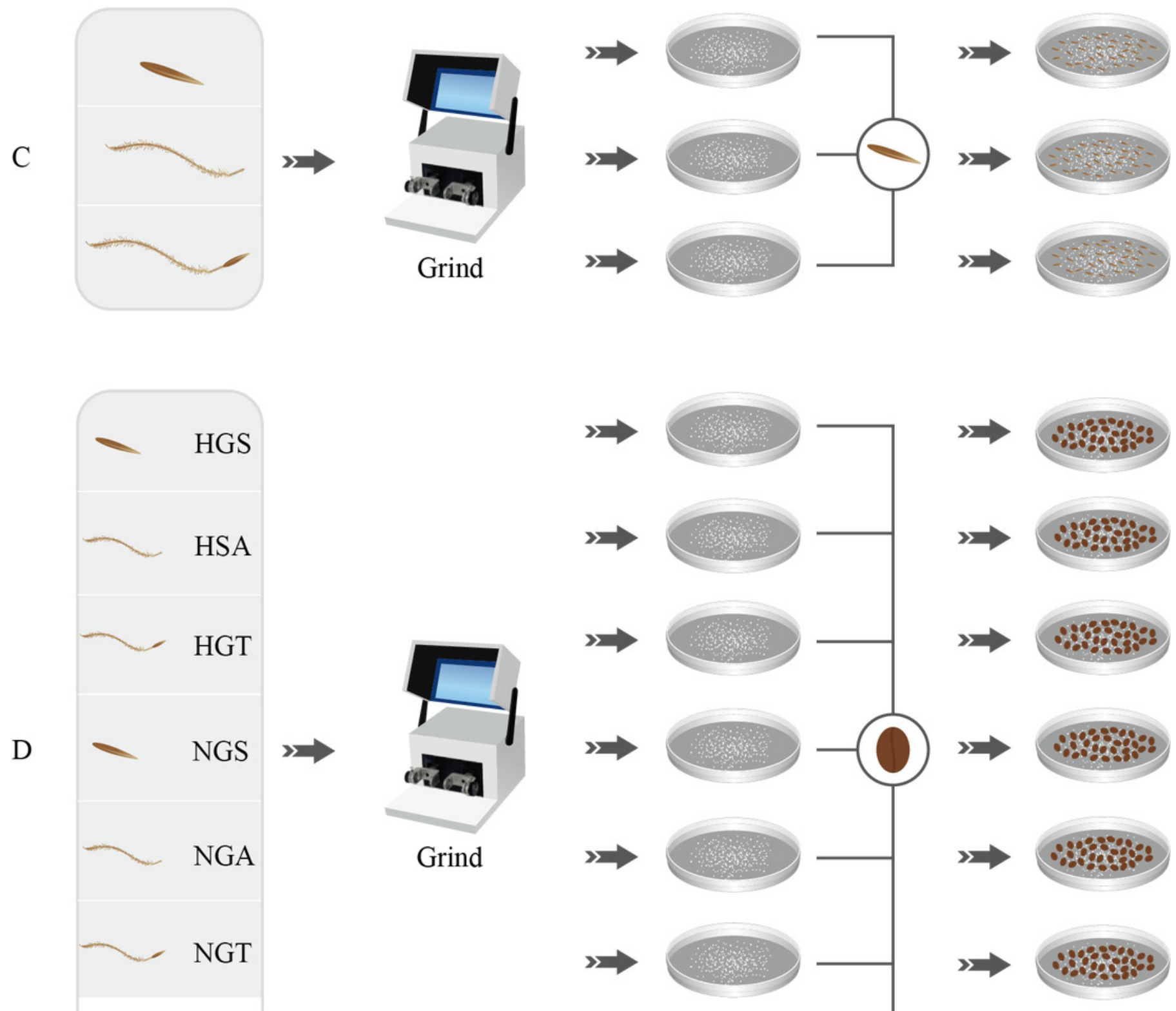
Figure 2

Seed characteristics of Stipa breviflora. 

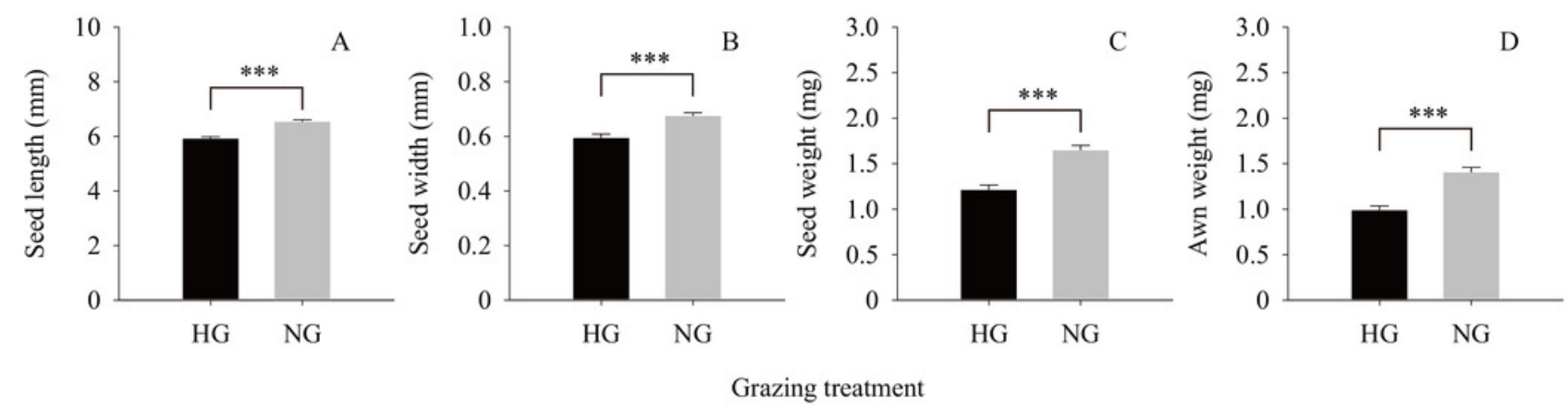

Grazing treatment 
Figure 3

Characteristics of seed germination of Stipa breviflora. 


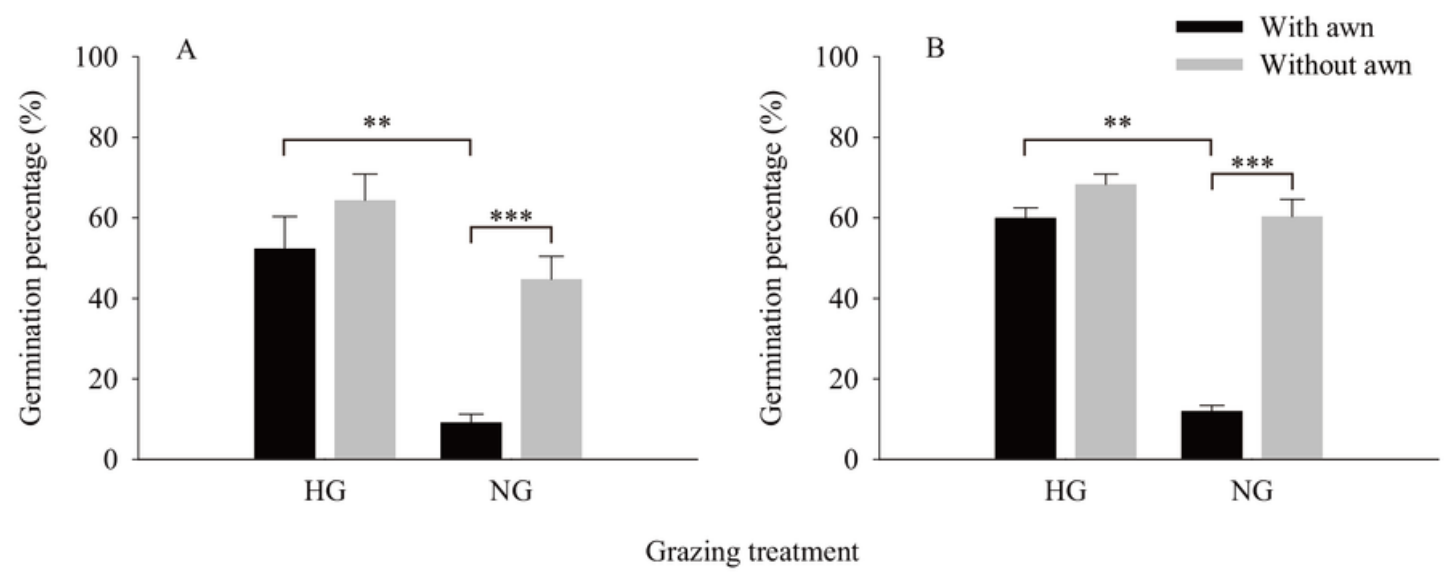




\section{Figure 4}

Effect of Stipa breviflora additives on the seed germination of Stipa breviflora.

The seed germination rate of Stipa breviflora treated with heavy grazing after adding nongrazing seeds (NGSs), awns (NGAs) and seeds + awns (NGTs) was studied. The germination rate of Stipa breviflora seeds (HGSs), awns (HGAs) and seeds + awns (HGTs) without grazing was studied. CK denotes the control. Different lowercase letters indicate significant differences, $\mathrm{P}<0.05$. 

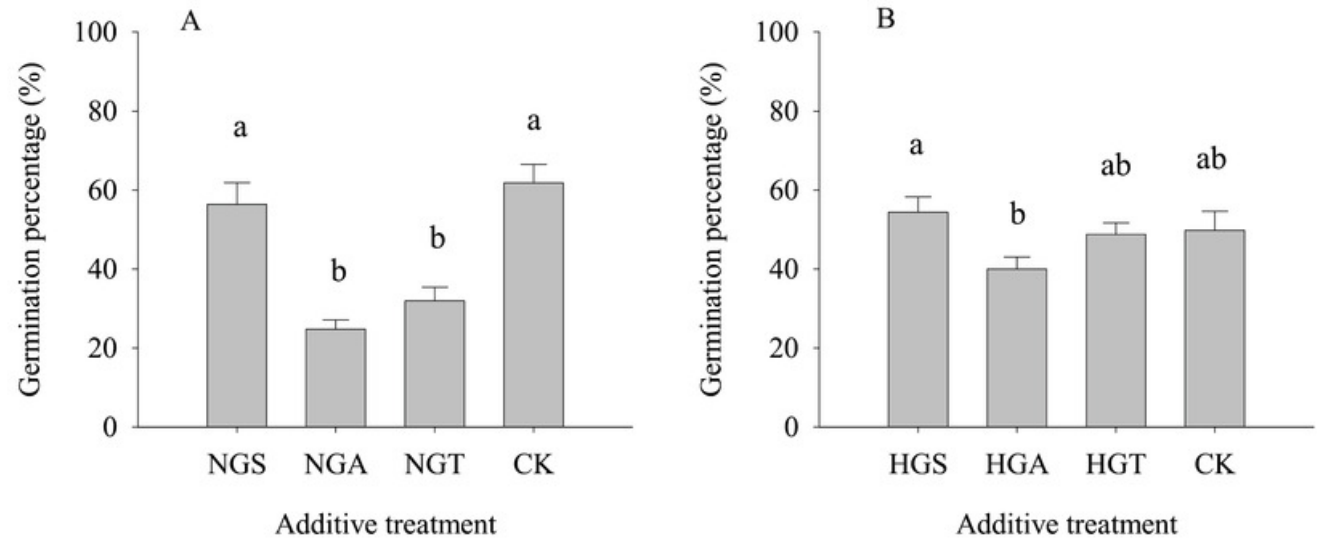


\section{Figure 5}

Effect of Stipa breviflora additives on the seed germination of Chinese cabbage.

HGS, HGA and HST indicate the seed, awn and seed + awn of Stipa breviflora under heavy grazing. NGS, NGA, and NGT indicate the seed, awn and seed + awn of Stipa breviflora under no grazing. CK was treated for contrast. Different lowercase letters indicate significant differences, $\mathrm{P}<0.05$. 


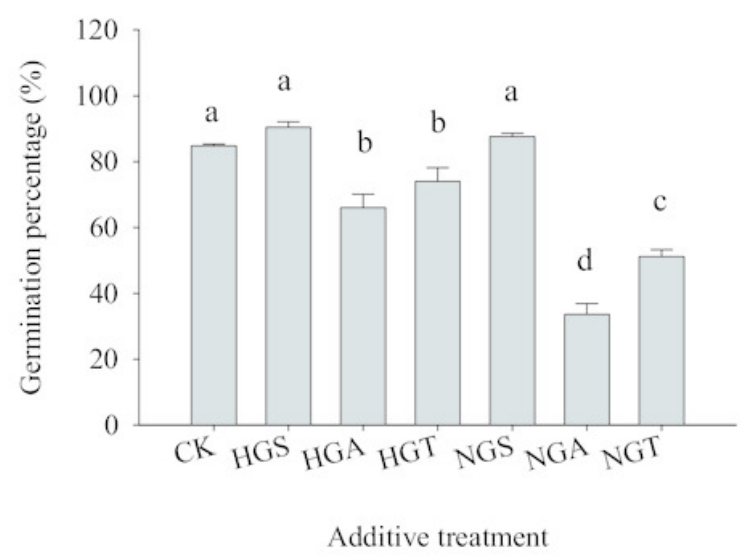

Peer] reviewing PDF | (2018:10:32087:2:0:NEW 31 Jan 2019) 


\section{Figure 6}

Population dynamics of the Stipa breviflora population.

The shadow depicts the standard error. * denotes $\mathrm{P}<0.05 ; * *$ indicates $\mathrm{P}<0.01$. HG and NG represent heavy grazing and no grazing, respectively. 


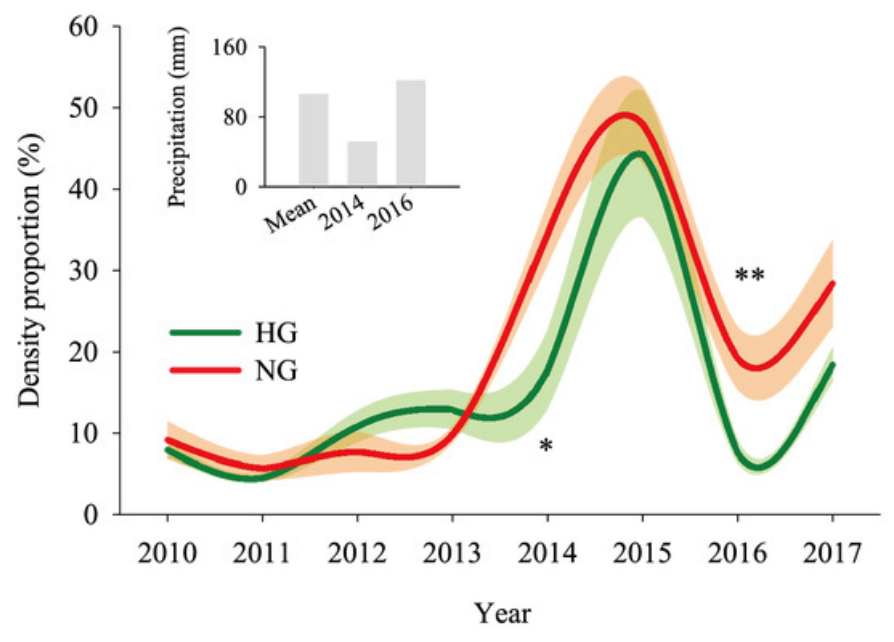




\section{Figure 7}

Population density characteristics of Stipa breviflora at different growth stages.

A, B and C are 2015, 2016 and 2017, respectively. ** indicates $P<0.01 . ~ H G$ and NG represent heavy grazing and no grazing, respectively. 

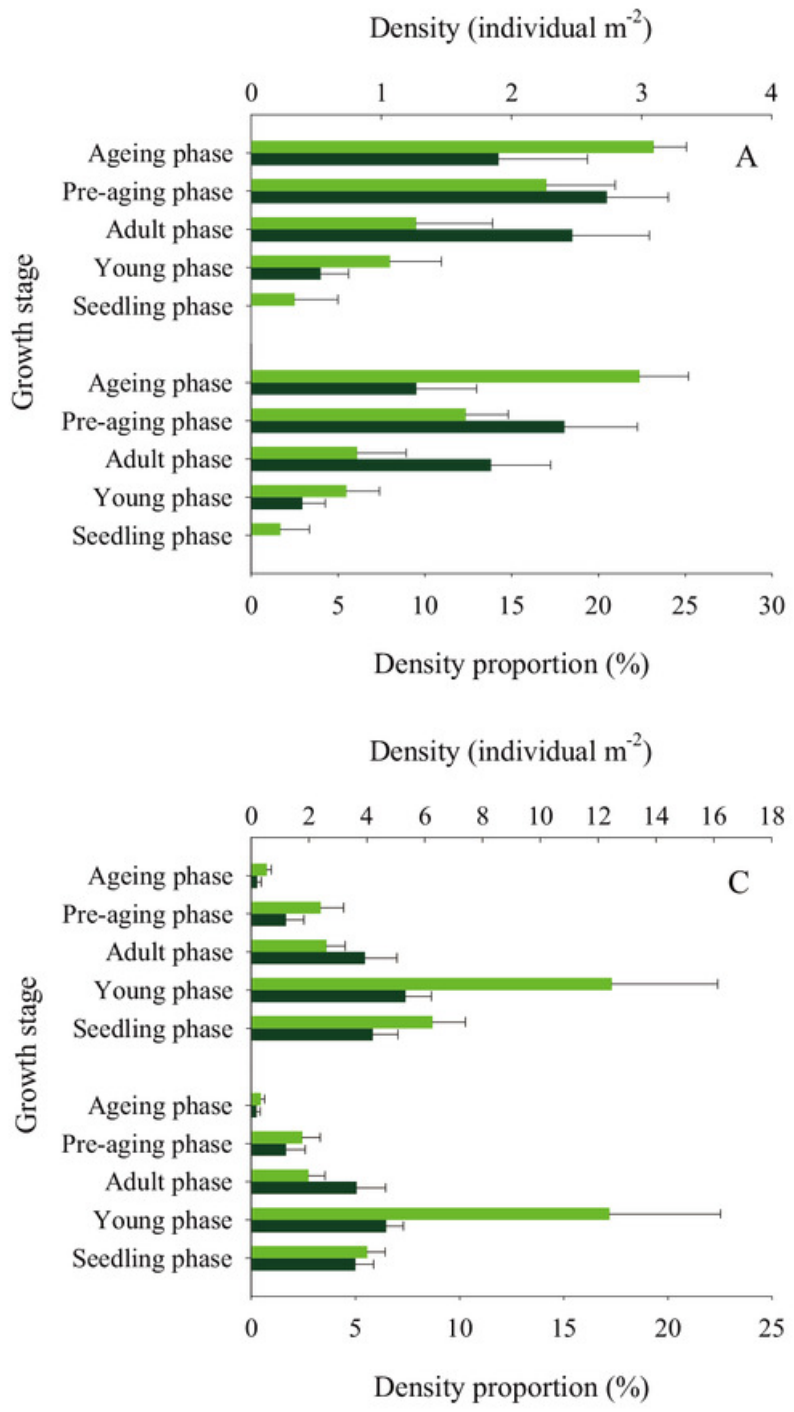

Density proportion (\%)

Pre-aging phase

Adult phase

Young phase

Ageing phase

re-aging phase

Young phase

Seedling phase

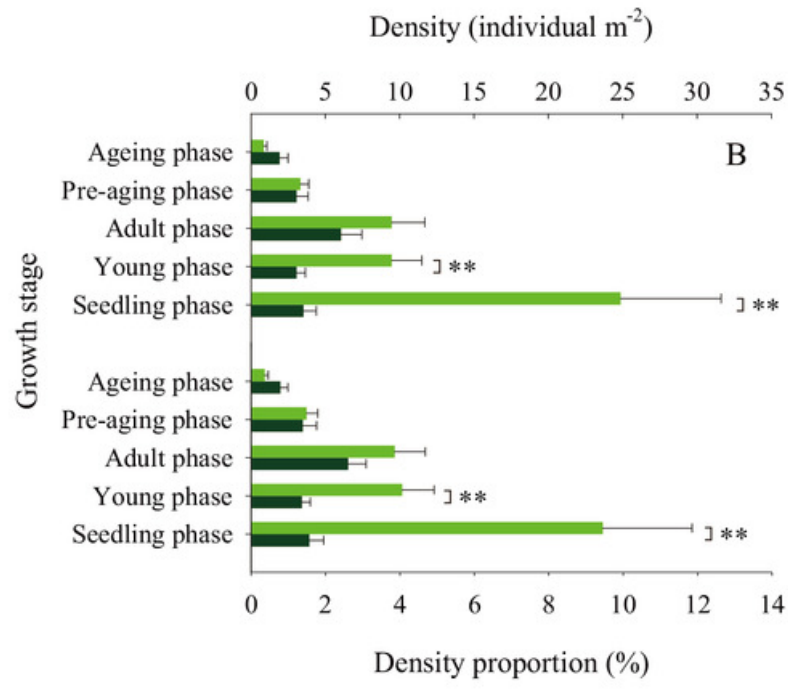

